\title{
Feasibility Study of Case-Finding for Breast Cancer by Community Health Workers in Rural Bangladesh
}

\author{
Touhidul Imran Chowdhury ${ }^{1}$, Richard Reed Love ${ }^{2 *}$, Mohammad Touhidul Imran \\ Chowdhury', Abu Saeem Artif ${ }^{1}$, Hasib Ahsan', Anwarul Mamun', Tahmina \\ Khanam$^{1}$, James Woods ${ }^{2}$, Reza Salim²
}

\begin{abstract}
Background: Mortality from breast cancer is high in low- and middle-income countries, in part because most patients have advanced stage disease when first diagnosed. Case-finding may be one approach to changing this situation. Materials and Methods: We conducted a pilot study to explore the feasibility of population-based case finding for breast cancer by community health workers (CHWs) using different data collection methods and approaches to management of women found to have breast abnormalities. After training $8 \mathrm{CHWs}$ in breast problem recognition, manual paper data collection and operation of a cell-phone software platform for reporting demographic, history and physical finding information, these CHWs visited 3150 women >age 18 and over they could find-- from 2356 households in 8 villages in rural Bangladesh. By 4 random assignments of villages, data were collected manually (Group 1), or with the cell-phone program alone (Group 2) or with management algorithms (Groups 3 and 4), and women adjudged to have a serious breast problem were shown a motivational video (Group 3), or navigated/accompanied to a breast problem center for evaluation (Group 4). Results: Only three visited women refused evaluation. The manual data acquisition group (1) had missing data in $80 \%$ of cases, and took an average of 5 minutes longer to acquire, versus no missing data in the cell phone-reporting groups (2,3 and 4). One woman was identified with stage III breast cancer, and was appropriately treated. Conclusions: Among very poor rural Bangladeshi women, there was very limited reluctance to undergo breast evaluation. The estimated rarity of clinical breast cancer is supported by these population-based findings. The feasibility and efficient use of mobile technology in this setting is supported. Successor studies may most appropriately be trials focusing on improving the suggested benefits of motivation and navigation, on increasing the numbers of cases found, and on stage of disease at diagnosis as the primary endpoint.
\end{abstract}

Keywords: Case-finding - breast cancer - cell-phone data collection

Asian Pac J Cancer Prev, 16 (17), 7853-7857

\section{Introduction}

The majority of global annual new breast cancer cases now occurs among poor women who live in low- and middle-income countries (LMICs) (Mathers and Loncar, 2006). While cause-specific mortality data are sparse, estimates are that while the mortality: incidence ratios for breast cancer in high-income countries are approaching 0.2 ; in low and middle income countries like Bangladesh this figure is 0.8 (Economist Intelligence Unit, 2009). The explanations for such dramatic differences are multiple, but from our experience in Bangladesh can be grouped under health system dysfunction and weaknesses, human rights shortcomings and mal-governance (Story et al, 2012). In Bangladesh, as is reported in many other LMICs, a high fraction of women with breast cancer is first seen when the disease is in advanced stages, for which curative treatment in any country is unlikely (Schwartzman, 2001; Malik, 2002; Omar et al., 2003; Story et al., 2012). In our work over the last several years exploring how to address this relatively rare disease with extremely poor outcomes in rural Bangladesh, women have expressed to our caregivers that they have realized for years to months that something was wrong in their breasts, but that they had no viable choices for how to address their problems (Story et al., 2012). Cancer registry data from Kolkata (next door to Bangladesh and part of "greater" Bengal) suggest that the age- corrected annual incidence is approximately $25 / 100,000 /$ year; with the broad-based age structure of the Bangladeshi population this means that among adult women perhaps 1 in 4000 each year might develop this malignancy (Sen et al., 2002). In these circumstances we began thinking about how we could develop a sustainable model system where health providers were pro-active in seeking out women with serious breast changes or problems, and facilitating their diagnosis and complete care. We surmised that our model would have to deal with the major underlying issues noted above, as well as being 
not vertical (focusing only on breast cancer) but horizontal to be scalable in addressing other prevalent serious health problems in rural Bangladesh- gastrointestinal infectious conditions, uterine cervical cancer, head and neck cancers, hypertension, chronic respiratory disease and diabetes (Alwan et al., 2011).

We report here the results of a population-based feasibility study of case-finding for breast cancer among very poor rural Bangladeshi women. Our data highlight the potential of mobile technology, case-finding and assistance in seeking care or navigation for addressing a rare disease like breast cancer where it is highly fatal.

\section{Materials and Methods}

\section{An m-Power community health worker model}

In many LMICs there are significant shortages of trained health professionals and community health workers (CHWs) with very limited training provide basic primary health care (mHealth for Development, 2010). Their usual limited paper record and data collection efforts are inefficient and the data are of uncertain value. m-Power has pioneered a CHW-led mHealth model, in which trained CHWs collect patients' health-related data using inexpensive cell phones loaded with sophisticated applications based on Java -J2ME- software. These data are digitized on a real-time basis and sent to a server through general packet radio service connectivity to create a comprehensive and secure electronic database. Decision-makers and doctors view the processed realtime information and Electronic Medical Record (EMR) in different interactive web-interfaces (DeRenzi et al, 2011). Such a technology-loops model we believe can extend the reach of and increase the efficient and more successful use of many specific limited health professional facilities and services in LMICs. An important part of this model is having health facilities which respond to CHWs' referrals and requests and actively involve these providers in the full spectrum of health care. For CHWs and weak health systems such a model provides an opportunity for CHWs as local health micro-entrepreneurs. We have successfully tested this model in Egypt, Botswana and Bangladesh, to provide a greater portfolio of healthcare services including maternal and child health, care for some non-communicable diseases, and case- finding for cervical cancer (Ghose, 2010; McWilliams, 2010). Such a model allows addressing the multiplicity of barriers that poor people face in obtaining health care, with the CHWs coming to the populations in need of service.

\section{Patients}

There is no data-base of households, "addresses" and citizens in Bangladesh. The last country-wide census was a decade ago, the population has been growing rapidly, and the ravages of typhoons particularly in the southwest rural area of the country in which this work took place, all contribute to a situation where testing a population-based sample required that we define that sample. We selected 8 similarly sized villages from among 138 in one geographic sub-district with limited major industry employment opportunities, in the central area of the Khulna division of the country where our non-governmental organization, Amader Gram, part of which is an information technology training organization, is known and trusted. . We paired villages into four groups we estimated to be of equal sizes and randomized these following which community health workers from these villages then mapped and registered 3658 households in the 8 villages, which reported 4649 women age 18 or over.

\section{Project administrative approval}

Individual community health workers in Bangladesh are usually fractionally government-employed, and otherwise self-employed. This project was approved as a quality of services investigation by the Khulna division of health department. That is what was appropriate in Bangladesh in such circumstances. The authorities in the division determined that it was within their prerogatives to make decisions about this project and did not feel that permission and review by the central Bangladeshi Medical Research Council which conducts combined scientific and ethical reviews of medical research in the country, was necessary. We received permission from the Divisional health authorities to: $i$ ) Train a group of 8 community health workers (CHWs) who lived and worked in the villages we selected for study, in breast health, breast examination and recognition of serious breast problems. ii) Have these CHWs visit and record, using village maps and assigning identification numbers, all households in each selected village and on a subsequent visit, interview and examine as many women in these villages as possible to collect demographic data and then specific data about breast problems, and to examine the breasts of all consenting women. Women provided informed consent at the time of this second visit, either by providing a signature, a thumb-print, or verbal consent which was recorded on a cell phone. iii) Have these $\mathrm{CHWs}$ provide the paper collected and cell-phone recorded data submitted to m-Power and a secure Amader Gram server.

\section{Design}

We conducted a pilot randomized population-based study to determine: $i$ ) Feasibility of serious breast problem case-finding by community health workers using either paper or cellphone recording of basic individual patient data; and ii) Feasibility of a motivational video and community health worker navigation of women found with serious breast problems, as interventions to get patients into medical care for those problems.

We made no power calculations for our sample size to address the first objective

A priori, we estimated that the numbers of women with serious breast problems found in the entire sample were likely to be small and that we would have little power to demonstrate differences for the interventions evaluated (\#2). However we could not know apriori whether the CHWs would in fact only identify "serious" breast problems.

After randomization, the mapping and registration of women in the 8 villages and four village groups, which were thought to be of similar sizes, provided unexpectedly estimates of $1337,711,920$, and 1681 potential women 
in the four groups.

\section{Community health worker training}

We conducted a two day training course for 8 community health workers of similar ages and educational experience about breast problems covering: Breast anatomy and physiology, common pathologic conditions, and cancer: presentations, diagnosis, treatments and curability. We further addressed barriers to seeking care with role playing for assistance by the community health workers with the perceived commonest issues: poverty, husband or mother-in-law proscription, travel/religious proscription, and child care. Finally we provided technical training, including review of all data items, and definitions in the use of the cell phone software applications and the household survey and the subsequent case finding visits. We emphasized completeness of data collection and working to detail. Demonstration training was also done using the different tools and interventions. The training emphasized that the most important abnormal findings on examination to be sought were: 1 . A distinct mass in one breast; 2. Skin or nipple changes in one breast; and 3. An area $>3 \mathrm{~cm}$ in diameter in one breast which was different from the tissue in the mirror image area of the opposite breast.

\section{Interventions}

All women visited were given a short leaflet about breast cancer signs and symptoms and where to go for care, and any women who were found to have a serious breast problems or a suspicious abnormality in one breast were given a referral card about the Amader Gram Breast Center in Khulna. Using a random number generator we randomly assigned eight villages into four intervention groups:

Group 1:Manual data collection and recording on paper forms of: $i$ ) Demographic and socioeconomic data: age, education, marriage status, number of children, occupation of household head, monthly expenditure. ii) Breast problems: breast mass in the past, a difference in one breast compared to the other and duration of this difference. iii) Examination findings of mass (breasts or axillae), skin or nipple changes or tissue differences $>3 \mathrm{~cm}$ in one breast. $i v$ ) Time taken to acquire information.

Group 2: Data collection and recording in cell-phone software program of the same four types of data as recorded with manual data collection.

Group 3: Data collection and recording in cell-phone software program with showing of a motivational video to any woman identified as having a serious breast problem. We created a short 3 minute testimonial video in which 3 patients with serious breast problems and breast cancer talked about their personal experiences in getting successful treatment from the Amader Gram Breast Center. The video was designed to address the widespread suspicion of medical systems and perceived uselessness of treatment for breast cancer among Bangladeshi women.

Group 4: Data collection and recording in cell-phone software program with navigation for any woman found to have a serious breast problem. To address several cultural and social barriers which prevent women from seeking care for serious health problems, we arranged this intervention: that when a community health worker found a woman with a serious breast problem, she would talk to her about going to the Amader Gram Breast Center in Khulna, hear her concerns about why and when and whether she would go, and then seek to address those concerns, arrange the appointment time, and most specifically in agreeing that she -the community health worker-- would travel with the affected woman to Khulna to the center and the expenses for this trip would be paid for.

\section{Data management and analysis}

An investigator reviewed $5 \%$ of all cases and data with CHWs.

All the data were analyzed in Microsoft Excel (version 2010) and STATA (College Station, Texas, USA).

\section{Results}

\section{Participants}

Sample creation: With village household mapping, 4,649 potential women age 18 or over were identified from 3,658 household visits in 127 community health worker work-days. Subsequently, 3,147 (67.7\%) of these women were examined in visiting 2,356 (64.4\%) households over 155 community health worker workdays. Following from the post- randomization different numbers of women mapped in the four groups -1337 , 711,920 , and 1681 , the numbers of women examined varied in the four groups-1118 (83\%), $334(47 \%), 620$ $(67 \%)$ and $1075(64 \%)$. Beyond the unexpected possibly available dis-similar numbers of women mapped from village pairs thought to be of equal population sizes, these extreme differences in examined group numbers occurred because of uncontrollable absence of a $\mathrm{CHW}$ for the second village group home visits, monsoon rain flooding differentially affecting the second, third, and part of four village groups. Only 3 identified women, at home when the community health worker visited, refused to participate in the study. 1499 women $(32.3 \%)$ of women identified by household mapping were not available at the time of the case-finding visits.

Demographic and socioeconomic characteristics of women (Table 1)

Of particular note: $21 \%$ of the study women were under age 25 . Half of the average 5 person households reported having less than \$3 to spend per day; 93\% had less than $\$ 5$ for the entire household.

There were some differences among the 4 randomized village groups: in age distribution and particularly in reported income with group 1 reported higher levels than the other groups.. Manual (Group 1) versus cell-phone software data collection (Groups 2, 3 and 4)

In 1118 women (Group 1) the data were collected and record manually on paper forms. In the three interventions arms (Groups 2,3 and 4) data were collected and recorded on a cell phone software program in 2029 women. The interviewed women voiced no reluctance to provide their information which they could see was being recorded in a cell phone. In the cell phone software data collection cases there were 405 errors in data entry subsequently 
Table 1. Socio-demographic Characteristics of Women Studied

\begin{tabular}{|c|c|c|c|c|c|}
\hline Indicators & $\begin{array}{c}\text { All, \% } \\
(\mathrm{N}=3147)\end{array}$ & $\begin{array}{c}\text { Group 1, \% } \\
(\mathrm{N}=1118)\end{array}$ & $\begin{array}{c}\text { Group 2,\% } \\
(\mathrm{N}=334)\end{array}$ & $\begin{array}{c}\text { Group 3, \% } \\
(\mathrm{N}=620)\end{array}$ & $\begin{array}{c}\text { Group 4, \% } \\
(\mathrm{N}=1075)\end{array}$ \\
\hline \multicolumn{6}{|c|}{ Number of women in each household } \\
\hline 2 or less & 95.49 & 96.2 & 97.43 & 97.23 & 91.11 \\
\hline $3-5$ & 4.23 & 3.5 & 2.57 & 2.77 & 8.09 \\
\hline$>5$ & 0.27 & 0.3 & 0 & 0 & 0.81 \\
\hline \multicolumn{6}{|l|}{ Age (years) } \\
\hline $18-24$ & 21.4 & 20.4 & 16.96 & 23 & 25.26 \\
\hline $25-34$ & 31.19 & 38.27 & 31.58 & 34.07 & 28.95 \\
\hline $35-44$ & 22.31 & 23.83 & 23.39 & 23.85 & 20.92 \\
\hline$>45$ & 23.02 & 17.51 & 28.07 & 19.08 & 24.87 \\
\hline \multicolumn{6}{|l|}{ Education } \\
\hline No formal education & 11.84 & 2.31 & 23.19 & 5.86 & 16.01 \\
\hline 1st-5th Grade & 27.16 & 33.27 & 27.25 & 19.37 & 28.76 \\
\hline 6th-8th Grade & 41.26 & 44.31 & 34.2 & 47.3 & 39.22 \\
\hline 9th-10th Grade & 12.88 & 12.99 & 8.41 & 18.02 & 12.09 \\
\hline 11th-12th Grade & 4.89 & 5.16 & 5.22 & 7.21 & 1.96 \\
\hline$>12$ th Grade & 1.98 & 1.96 & 1.74 & 2.25 & 1.96 \\
\hline \multicolumn{6}{|c|}{ Monthly Household Expenditure (BDT) ${ }^{\text {BDT }=\text { Bangladeshi taka. } 77 \text { BDT }=1 \text { US\$ }}$} \\
\hline$<3,000$ & 16.11 & 18.12 & 16.96 & 10.96 & 18.42 \\
\hline $3,001-6,000$ & 35.21 & 1.22 & 55.85 & 50.23 & 33.55 \\
\hline $6,001-9,000$ & 18.02 & 0.35 & 17.54 & 25.57 & 28.62 \\
\hline $9,001-12,000$ & 23.09 & 70.03 & 2.92 & 8.22 & 11.18 \\
\hline $12,000-15,000$ & 4.86 & 7.14 & 6.14 & 3.2 & 2.96 \\
\hline$>15,000$ & 2.7 & 3.14 & 0.58 & 1.83 & 5.26 \\
\hline \multicolumn{6}{|l|}{ Marital Status } \\
\hline Married & 91 & 95 & 91 & 90 & 89 \\
\hline Unmarried & 9 & 5 & 9 & 10 & 11 \\
\hline
\end{tabular}

identified (20\% of cases) and 0 cases ( $0 \%$ ) with omission of data [With the cell-phone application, every question had to be answered or exiting the case entry was impossible]. The cell phone errors were in patient and household identification. In contrast among the manual data collection cases, there were 503 errors in data entry subsequently identified ( $45 \%$ of cases) All of these errors concerned patient or household identification. 894 cases (80\%) had omission of data in the following categories: Age (4\%), Weight (11\%), Answer to the breast problemYes or No (3\%), Physical exam of breast (1\%), Physical exam of axillae (57\%). Manual data entry was reported to take an average of 13 minutes (10-15 minutes) while with cell-phone software data entry averaged 8 minutes (7-10 minutes).

Intervention with motivational video (group 3) or navigation (group 4):

Among all examined subjects, ten women were identified with suspicious breast abnormalities. Four of these women were from the manual data collection-only group (\#1), and of these one followed up and visited the Amader Gram Breast Center and was found to have no serious problem. Six women from the motivational video (group 3) and navigational (group 4) interventional groups were found to have suspicious breast abnormalities, and four of these were seen in the Amader Gram Breast Center where one was found to have stage III breast cancer. Among the five women not specifically evaluated at the Amader Gram Breast Center, we have been able to determine by having CHWs return and examine and question these women three months later, that all of the five are very unlikely to have a serious breast problem. Among 3147 women if the incidence rate is $25 / 100,000 /$ year, 0.79 incident cases might be expected to be found.

\section{Discussion}

In this population-based study, we were unable to do specific case-finding among $32 \%$ of the women we had identified as living in the households in our research population. This situation means we may have missed some women with serious breast problems-breast cancer. The only reassurances we can provide that our results and conclusions about actual prevalent breast cancer cases in our study population should not be called into question are that the major reasons these women were not investigated -- absence of a CHW for visits, and monsoon floodingappear not to be related to willingness to be examined or likelihood of having a serious breast problem. We have no reasons to believe that demographically these women were significantly different from those we did examine.

While the same CHWs conducted the data collection using both manual and cell phone methods and, as indicated, interventions with motivating video or navigation, we have no reasons to believe that they were invested in seeing particular approaches prove more successful, and believe they were making good faith efforts to do the individual interventions for each group as best they could. In training we simply stated that this was how the data would be collected in different villages.

The findings from this study are of practical 
importance in addressing the challenges of breast cancer in this low-income country. First cell-phone software case-finding data entry can be successfully accomplishedour rural women were not reluctant or suspicious of the process of their information being recorded on a cell phone-- and in our experience cell phone data entry is demonstrably better than manual entry in researcher time per case and completeness of data obtained. The inaccuracy of patient and household identification problem, which was significantly less with the cell phone entry tool, is one we can partially address by having electronically submitted information immediately checked against the available data base. Second, contrary to our expectations, the unwillingness of women in this $60 \%$ Muslim Khulna division society, to report about their breast symptoms, and to be examined, was very low. Third, in a population-based sample, we have provided data supporting the expected low rate of breast cancer in this country. While we included as $21 \%$ of our population, women under age 25 , which might be expected to lower the rate of breast cancer cases found, we found only one incident/prevalent case among 3147 women, when as indicated the incident cases might be expected to be 0.78 . This finding must be strongly considered in thinking about any future strategies directed to this disease and the "late" presentation problem in Bangladesh. It certainly reinforces arguments made about the inapplicability of screening strategies from high incidence-high-income countries to countries with low incidence like Bangladesh (Formenti et al., 2012). Finally, our data support the feasibility of using a motivational video and importantly navigation whereby a community health worker accompanies a woman with a suspected serious breast problem, to a specialty health care facility, can get women into care. More importantly it suggests that this approach is culturally acceptable. We need to understand how to better strengthen this kind of intervention.

A more general issue raised by our study and results concerns follow-up investigation. We suggest that we have adequately proven principle that community health workers can more efficiently use cell-phone software for case-finding, with greater accuracy, than paper manualrecorded data. We believe that the major time/effort and cost issues outweigh the need to test this technological applications question further. Successor studies may most appropriately be trials focusing on motivation and navigation which from our limited experience appears feasible, on increasing the numbers of cases found, and on stage of disease at diagnosis as the primary endpoint.

In conclusion we have demonstrated the feasibility of population based case finding for breast cancer in a low incidence low income country setting. Additionally we have shown that cell-phone technology can be a useful and efficient tool in case-finding for breast cancer in such a setting, and developed strongly suggestive data that the estimated low population incidence rates for breast cancer in Bangladesh are correct. Finally, contrary to expectations we have found that almost all poor rural women in this dominantly Muslim country were very willing to answer questions about breast problems and be examined.

\section{Acknowledgements}

The following individuals provided important assistance in this project: Meer Tanvir Hossain Tamal, Khan Zahid Hossain, Dr. Syed Mozammel Hossain, Dr. Moslema Parvin Akhi, Michael Scahill, Dr. Shamiul Haque and Rifat Hasan

\section{References}

Alwan A, Armstrong T, Cowan C, et al (2011). Noncommunicable diseases country profiles. World Health Organization. Retrieved January 03, 2012, from http:// whqlibdoc.who.int/publications/2011/9789241502283_eng. pdf

DeRenzi B, Borriello G., Jackson J, et al (2011). Mobile Phone Tools for Field-Based Health care Workers in Low-Income Countries. Mount Sinai J Med, 78, 406-18

Economist Intelligence Unit, Breakaway (2009). The global burden of cancer-challenges and opportunities. The Economist.

Formenti SC, Arslan AA, Love SM, (2012). Global breast cancer: the lessons to bring home. Int J Breast Cancer.

Ghose S. (2010). The use of mobile (Cellular) oral telemedicine in botswana. Mobile Communication Technol Development (M4D), 336-7.

Malik I A (2002). Clinico-pathological features of breast cancer in Pakistan. J Pakistan Med Assoc, 53, 100-4.

Mathers C D, Loncar D, (2006). Projections of global mortality and burden of disease from 2002 to 2030. PLoS Med, 3, 442 .

McWilliams J, (2010). Connecting cell phones with medicine in Botswana. Penn Current. Retrieved November 25, 2011, from http://www.upenn.edu/pennnews/current/node/3215

mHealth for Development (2010). The Opportunity of Mobile Technology for Healthcare in the Developing World. United Nations Foundation \& Vodafone Foundation.

Omar S, Kahled H, Gaafar R, et al (2003). Breast cancer in Egypt: A review of disease presentation and detection strategies. East Med Health J, 9, 448-63

Schwartzmann G, (2001). Breast Cancer in South Africa: Challenges to improve early detection and medical management of a public health problem. J Clin Oncol, 19, 118-24.

Sen U, Sankaranarayanan R, Mandal S, et al (2002). Cancer patterns in Eastern India: the first report of the Kolkata Cancer Registry, Int J Cancer 100: 86-91.

Story H L, Love R R, Salim R, et al (2012). Improving outcomes from breast cancer in a low-income country: lessons from Bangladesh. Int J Breast Cancer. 